\title{
High Dose-Volume SBRT Following TACE Improves Clinical Outcomes of Patients with Unresectable Hepatocellular Carcinoma
}

\author{
Nanbao Zhong*, Guangming Lv*, Zhonghua Chen\# \\ Tumor Radiotherapy Center, Fuzhou General Hospital, Fuzhou, China \\ Email: "zyczhonghua@163.com
}

Received 9 January 2016; accepted 26 February 2016; published 29 February 2016

Copyright (C) 2016 by authors and Scientific Research Publishing Inc.

This work is licensed under the Creative Commons Attribution International License (CC BY). http://creativecommons.org/licenses/by/4.0/

(c) (i) Open Access

\section{Abstract}

This study aimed to investigate the dose-volume effects of $\gamma$-ray stereotactic body radiotherapy (SBRT) on clinical outcomes of patients with huge-size $(\geq 10 \mathrm{~cm})$ unresectable hepatocellular carcinoma (HCC). A total of 59 patients with huge-size unresectable HCC were treated with SBRT following TACE between May 2006 and Dec. 2009. The analyzed parameters included fractional dose, marginal dose, maximal dose, and mean dose that the target received, as well as percentages of tumor volume encompassed by $60 \%\left(\mathrm{P}_{60}\right), 70 \%\left(\mathrm{P}_{70}\right)$, and $80 \%\left(\mathrm{P}_{80}\right)$ of isodose curves in entire tumor. The clinical outcomes included objective response rate (ORR), disease-free survival (DFS), overall survival (OS), and adverse event (AE). During median follow-up of 18.4 months, $81.4 \%$ of ORR (8.5\% CR and $72.9 \%$ PR) was achieved, higher than $28.9 \%$ of ORR recently reported for TACE alone. 1- and 3-year DFS rates were $31.1 \%$ and $2.6 \%$ with median DFS of 8.7 months; $1-$, 3-, and 5 -year OS rates were $46.5 \%, 13.7 \%$, and $2.9 \%$, with median OS of 11.8 months. $P_{70}$ was the only factor significantly correlating to DFS $(P=0.009)$ and $O S(P=0.01)$. Neither severe radiation-related liver disease nor > grade $3 \mathrm{AE}$ was observed. In conclusion, SBRT was a safe and effective option for treatment of huge-size unresectable HCC. $P_{70}$ represented a parameter for predicting DFS and $O S$, and high dose-volume (e.g., $P_{70}$ ) might be required to achieve improved clinical outcomes of patients with this type of HCC.

\section{Keywords}

Stereotactic Body Radiotherapy, Dose-Volume Parameter, Disease-Free Survival, Overall Survival, Unresectable Hepatocellular Carcinoma

\footnotetext{
*These authors contributed equally to this work.

"Corresponding author.
}

How to cite this paper: Zhong, N.B., Lv, G.M. and Chen, Z.H. (2016) High Dose-Volume SBRT Following TACE Improves Clinical Outcomes of Patients with Unresectable Hepatocellular Carcinoma. Journal of Cancer Therapy, 7, 152-161. 


\section{Introduction}

Hepatocellular carcinoma (HCC) is one of the most common cancers worldwide, particularly in developing countries [1]. Recently, its incidence has been increasing in North America [2]. HCC is also the third leading cause of cancer-related deaths [3]. The 5-year overall survival rate of HCC is only 3\% - 5\% [4]. There are 10\% $20 \%$ of patients with newly diagnosed HCC that are larger than $10 \mathrm{~cm}$ in diameter [5]. To date, treatment option for huge-size $(\geq 10 \mathrm{~cm})$ HCC is very limited. PEI, RFA, and liver transplantation are usually not appropriate modalities for the treatment of patients with such large HCC [6]-[8]. Transarterial chemoembolization (TACE) alone is also unsatisfactory in treatment of large tumors [9]. Currently, hepatectomy is considered as the best option for the treatment of HCC [10]. However, a high recurrence rate $(50 \%-70 \%)$ remains a major issue after curative tumor resection [11] [12]. Furthermore, many cases of huge-size HCC are unresectable [13] [14]. Therefore, effective treatments for such a type of HCC are desperately needed.

In earlier radiotherapy, the whole liver was irradiated to treat HCC. The dose for the whole-liver radiotherapy that could be tolerated was too low to affect tumor [15]-[17]. As consequence, radiotherapy had not been widely used in the management of patients with HCC. With the recent advances in science and technology, radiotherapy had emerged as a potentially curative option in the treatment of HCC [18]. Among many others, stereotactic body radiotherapy (SBRT, also known as hypo-fractionated radiotherapy) represented one of the most advanced technologies in radiotherapy. Several studies had demonstrated that SBRT had the significant therapeutic effects on HCC [19]-[25]. In this context, a phase 2 trial was currently ongoing to evaluate the effectiveness and adverse event of SBRT in the patients with unresectable HCC who had solitary $3 \mathrm{~cm}$ or less size HCC without extrahepatic lesion and vascular involvement (https://clinicaltrials.gov/ct2/show/NCT01910909). Moreover, since August 2014, Radiation Therapy Oncology Group (RTOG) has been conducting a phase 3 randomized multicenter trial (RTOG 1112) to determine if SBRT followed by Sorafenib improve overall survival in unresectable HCC patients, by comparing to Sorafenib alone

(https://www.rtog.org/ClinicalTrials/ProtocolTable/StudyDetails.aspx?study=1112). However, there were few reports on the role of SBRT in the treatment of huge-size HCC. Furthermore, as the dose-volume parameters of SBRT were entirely different from those of the conventional radiotherapy, it remained virtually unknown whether dose-volume parameters would impact on patient outcomes when SBRT was used to treat huge-size HCC. To this end, the present study was designed to investigate the effect of SBRT with high dose-volume parameters after incomplete TACE on the clinical outcomes of patients with huge-size unresectable HCC.

\section{Materials and Methods}

\subsection{Patient Eligibility}

This study was approved by the Ethics Committee of the Fuzhou General Hospital, and performed according to the Declaration of Helsinki. Written informed consent was obtained from each patient before treatment with TACE and SBRT. A total of 716 consecutive HCC patients were routinely treated with $\gamma$-ray SBRT as standard care between May 2006 and Dec. 2009 at the Tumor Radiotherapy Center, Fuzhou General Hospital, China. Among them, 59 patients were eligible for this study. The criteria for patients to be enrolled include: 1) unresectable HCC; 2) tumor size $\geq 10 \mathrm{~cm}$ in diameter; 3) Child-Pugh Class, A or B; 4) Eastern Cooperative Oncology Group Performance Status (ECOG PS), 0 - 2; 5) no extrahepatic metastasis; 6) SBRT post incomplete TACE for treatment; and 7) no history of liver radiotherapy. All patients had contrast-enhanced computed tomography (CT), magnetic resonance imaging (MRI), and/or positron emission tomography (PET) of abdomen. HCC was diagnosed by cytological/histological evidence $(n=51)$; one radiological image showing characteristic features of HCC, together with an elevated AFP level $(>400 \mathrm{ng} / \mathrm{ml}, \mathrm{n}=3)$; or at least two radiological images showing characteristic features of HCC $(n=5) .59$ patients were divided into group A $\left(\mathrm{P}_{70} \geq 60 \%, n=27\right)$ and B $\left(\mathrm{P}_{70}<\right.$ $60 \%, \mathrm{n}=32$ ). Characteristics of the patients were summarized in Table 1.

\subsection{Treatment}

TACE was carried out by infusion with the mixture of 5 - $10 \mathrm{ml}$ iodized oil (Lipiodol; Guerbet, Charles de Gaulle, France) and $1 \mathrm{mg} / \mathrm{kg}$ cisplatin (Dong-A Pharm. Co. Ltd., Seoul, Korea), followed by gelatin sponge cubes (Gelfoam; Upjohn, Kalamazoo, MI). Tumor feeding arteries were carefully selected for TACE, in order to preserve liver function the best. When there was an arterio-portal shunt, TACE was performed without Lipiodol 
Table 1. Characteristics of the patients $(n=59)$.

\begin{tabular}{|c|c|c|c|c|}
\hline \multirow{2}{*}{ Characteristic } & \multicolumn{2}{|c|}{ Group A } & \multicolumn{2}{|c|}{ Group B } \\
\hline & Value & No. of patient (\%) & Value & No. of patient (\%) \\
\hline \multicolumn{5}{|l|}{ Age, year } \\
\hline Range & $41-65$ & & $38-64$ & \\
\hline Mean & 53 & & 51 & \\
\hline \multicolumn{5}{|l|}{ Gender } \\
\hline Male & & $22(81.5)$ & & $26(81.3)$ \\
\hline Female & & $5(18.5)$ & & $6(18.7)$ \\
\hline \multicolumn{5}{|l|}{ ECOG PS } \\
\hline $\mathbf{0}$ & & $2(7.4)$ & & $3(9.4)$ \\
\hline 1 & & $20(74.1)$ & & 23 (71.9) \\
\hline 2 & & $5(18.5)$ & & $6(18.7)$ \\
\hline \multicolumn{5}{|l|}{ Child-Pugh } \\
\hline $\mathbf{A}$ & & $20(74.1)$ & & $24(75)$ \\
\hline $\mathbf{B}$ & & $7(25.9)$ & & $8(25)$ \\
\hline \multicolumn{5}{|l|}{ AFP (ng/mL) } \\
\hline$\geq \mathbf{4 0 0}$ & & $22(81.5)$ & & $25(78.1)$ \\
\hline$<400$ & & $5(18.5)$ & & 7 (21.9) \\
\hline HBsAg positive & & $21(77.8)$ & & 23 (71.9) \\
\hline Anti-HCV positive & & $3(11.1)$ & & $4(12.5)$ \\
\hline \multicolumn{5}{|l|}{$\mathrm{C} / \mathrm{h}$ confirmation } \\
\hline Yes & & 24 (88.9) & & $27(84.4)$ \\
\hline No & & $3(11.1)$ & & $5(15.6)$ \\
\hline
\end{tabular}

ECOG PS, Eastern Cooperative Oncology Group performance status; C/h, cytological/histological; AFP, alpha-fetoprotein; HBsAg, hepatitis B surface antigen.

to prevent severe damage of normal liver tissue.

SBRT was administrated using the total body $\gamma$-ray stereotactic radiotherapy system (OUR company, Shenzhen, China) 2 - 4 weeks after TACE. Briefly, patients were immobilized by vacuum cushions and underwent CT scan in supine or prostrate position. The CT data were then transferred to the SBRT Treatment Planning System (SGI, Southeast University, China). Body surface, tumor contour, and some important normal tissues were reconstructed to display three-dimensional (3D) representation based on cross-sectional imaging. Clinical target volume (CTV) is defined as the macroscopic volume of tumor. The planning target volume (PTV) was created by asymmetrically expanding the CTV by $0.5-1.0 \mathrm{~cm}$. The position, number, and size of focused fields were carefully selected to enhance the dose for PTV but minimize both the dose for normal tissues and irradiated tissue volumes. The dose-volume histogram (DVH) generated was then used to evaluate the treatment planning. Dose prescription was normalized at the $50 \%$ or $55 \%$ isodose curves encompassing $100 \%$ of PTV. Images were taken to verify the tumor localization and patient position before SBRT. Marginal dose and fractional dose determined dependently upon predicted toxicity of normal tissues and the function of reserved liver tissue. The marginal dose of $37.6 \pm 2.9$ Gy was delivered in 12 - 14 days with fractional dose of $2.8 \pm 0.2$ Gy and 6 fractions per week. All patients had one day of rest after every 6 consecutive fractions of treatment. The dose-volume parameters of tumors were shown in Table 2.

\subsection{Evaluation of Clinical Outcomes}

Complete blood counts (CBC) and liver function were assessed weekly during treatment. Tumor size within the 
Table 2. Dose-volume parameters.

\begin{tabular}{lcc}
\hline Variable & Group A & Group B \\
\hline PTV (cc) & $283-818$ & $292-823$ \\
Range & 574 & 581 \\
Median & & $34.7-40.5$ \\
Delivered dose dose (Gy) & $35.2-40.4$ & $59.7-68.5$ \\
Marginal & $61.8-67.1$ & $46.2-53.4$ \\
Maximal & $48.2-51.8$ & $2.6-3.0$ \\
Mean & $2.6-3.0$ & 100 \\
Fractional & & $67-89$ \\
Dose-volume, \% & 100 & $38-58$ \\
$P_{50}$ & $76-95$ & $15-34$ \\
$P_{60}$ & $60-72$ & $25-42$ \\
$P_{70}$ & & \\
$P_{80}$ & & \\
\hline
\end{tabular}

radiated field was measured based on CT and/or MRI scan 4 weeks after treatment completed followed by once for every 1 to 3 months afterwards. According to the Response Evaluation Criteria in Solid Tumors (RECIST), complete response (CR) was defined as disappearance of tumor, partial response (PR) as a $>30 \%$ decrease in tumor size, progressive disease (PD) as $>20 \%$ in-field tumor growth or new tumor appearance, and stable disease (SD) as neither PR nor PD criteria met. Objective response rate (ORR) was defined as a sum of CR and PR. Survival time was defined as a period from the date to start treatment to the date of death or for the last follow-up.

Acute and later toxicities were assessed using the National Cancer Institute (NCI) Common Toxicity Criteria Version 2.0 and the Late Radiation Morbidity Scoring Scheme of Radiation Therapy Oncology Group/European Organization for Research and Treatment of Cancer (RTOG/EORTC), respectively.

\subsection{Statistical Analysis}

Kaplan-Meier method was used to analyze overall survival (OS) rate. Survival curves were compared using the log-rank test. For multivariate analysis to evaluate the relation between the OS and various parameters, the stepwise procedure was performed using the Cox regression model. $\mathrm{P}<0.05$ was considered as statistically significant. All statistical analyses were conducted using the SPSS 16.0 (SPSS, Chicago, IL, USA) statistical software package.

\section{Results}

\subsection{Tumor Response}

Among 59 patients, 5 (8.5\%) and 43 (72.9\%) achieved CR and PR, respectively, yielding 81.4\% of ORR. In addition, 7 (11.9\%) patients had SD, while 4 (6.7\%) patients experienced PD. The median follow-up time was 18.4 months. Representative CT images before and after SBRT were shown in a patient who achieved a CR (Figure 1 ), in whom no tumor recurrence within the irradiated field was observed in 63 months after SBRT. Tumor responses are then compared between group $\mathrm{A}\left(\mathrm{P}_{70} \geq 60 \%\right)$ and $\mathrm{B}\left(\mathrm{P}_{70}<60 \%\right)$. As shown in Table 3 , 4 patients in group A achieved CR, while only one with a CR in group B. However, the number of patients with PR as well as ORR were similar between these two groups. These findings argue that SBRT following incomplete TACE is effective in the treatment of patients with huge-size unresectable HCC. They also raise a possibility that although there is no significant difference in ORR, $\mathrm{P}_{70} \geq 60 \%$ might yield higher CR than $\mathrm{P}_{70}<60 \%$. 

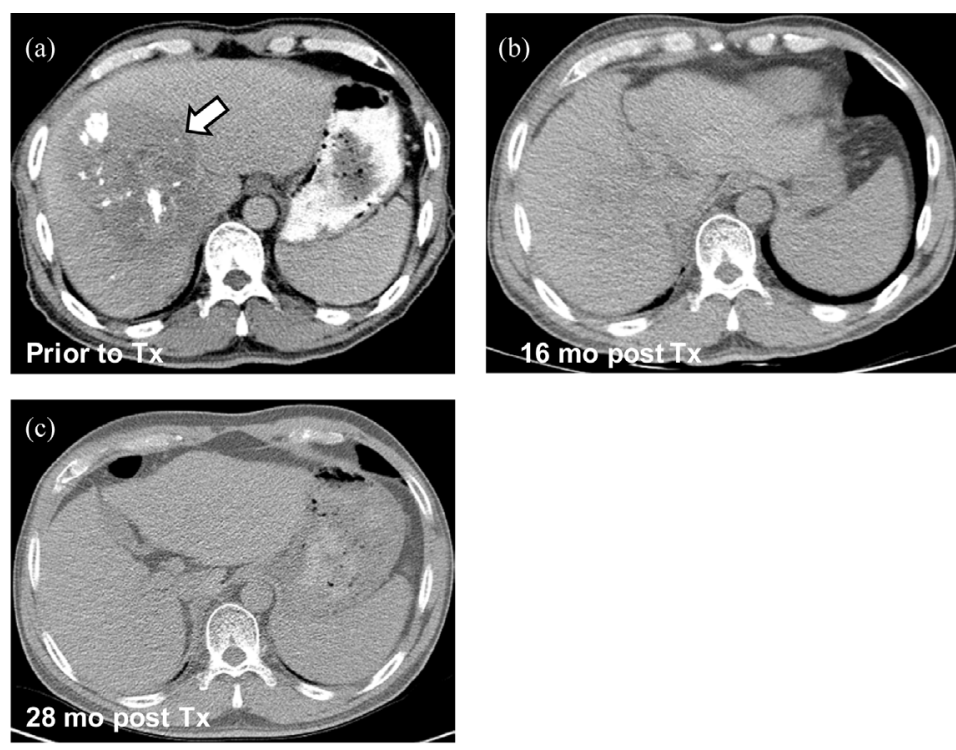

Figure 1. Representative images of abdominal CT scan in a male patient with hugesize HCC who achieved a CR after treated (Tx) with SBRT $(3.0 \mathrm{~Gy} / \mathrm{F} \times 13 \mathrm{~F} / 2 \mathrm{w})$ following TACE. The images were captured before SBRT (a), 16 months (mo, b), and 28 months after SBRT (c), respectively. Arrow indicates the huge-size HCC tumor.

Table 3. Tumor response after treated with SBRT following TACE.

\begin{tabular}{lccc}
\hline \multirow{2}{*}{ Response } & & No. of patient (\%) & Total \\
\cline { 2 - 4 } Complete response (CR) & Group A & Group B & $5(8.5)$ \\
Partial response (PR) & $4(14.8)$ & $1(3.1)$ & $43(72.9)$ \\
Stable disease (SD) & $21(77.8)$ & $22(68.8)$ & $7(11.9)$ \\
Progressive disease (PD) & $1(3.7)$ & $6(18.7)$ & $4(6.7)$ \\
Objective response (OR = CR + PR) & $1(3.7)$ & $3(9.4)$ & $48(81.4)$ \\
\hline
\end{tabular}

\subsection{Survival}

All of 59 patients were followed up for 4 to 70 months (median 18.4 months). Kaplan-Meier analysis revealed that 1- and 3-year DFS rates were 31.1\% and 2.6\%; 1-, 3-, and 5-year OS rates were 46.5\%, 13.7\%, and 2.9\%; median DFS (Figure 2(a)) and OS (Figure 2(b)) were 8.7 and 11.8 months, respectively. Cox regression multivariate analysis demonstrated that while marginal dose, maximal dose, mean dose, and P60 were not related with either DFS or OS, P70, P80, fractional dose, and Child-Pugh class (A versus B) were likely associated with DFS and OS. However, the likelihood of P80 ( $\mathrm{P}=0.054$ for DFS and 0.063 for OS), fractional dose $(\mathrm{P}=0.071$ for DFS and 0.076 for OS), and Child-Pugh class (P $=0.066$ for DFS and 0.068 for OS) were not statistically significant. Of note, P70 was the only factor significantly predicting both DFS $(\mathrm{P}=0.009)$ and OS $(\mathrm{P}=0.01) .1$ (51.6\%) and 3-year (5.4\%) DFS rates in group $\mathrm{A}\left(\mathrm{P}_{70} \geq 60 \%\right)$ were higher than those $(10.1 \%$ and $0 \%$, respectively) in group $\mathrm{B}\left(\mathrm{P}_{70}<60 \%\right)$. Consistently, 1- (69.7\%), 3- (23.6\%), and 5-year (6.1\%) OS rates in group A were also higher than those (23.2\%, 4.2\%, and 0\%, respectively) in group B. Median DFS (Figure 2(c)), and OS (Figure 2(d)) were 11.6 and 18.3 months for group A versus 7.1 and 9.6 months for group B. The findings indicate that SBRT following incomplete TACE improve survival of patients with huge-size unresectable HCC, and that the dose-volume parameter $\mathrm{P}_{70} \geq 60 \%$ is significantly better than $\mathrm{P}_{70}<60 \%$.

\subsection{Safety}

All of 59 patients completed the treatment with $\gamma$-ray SBRT following incomplete TACE. The maximal doses 


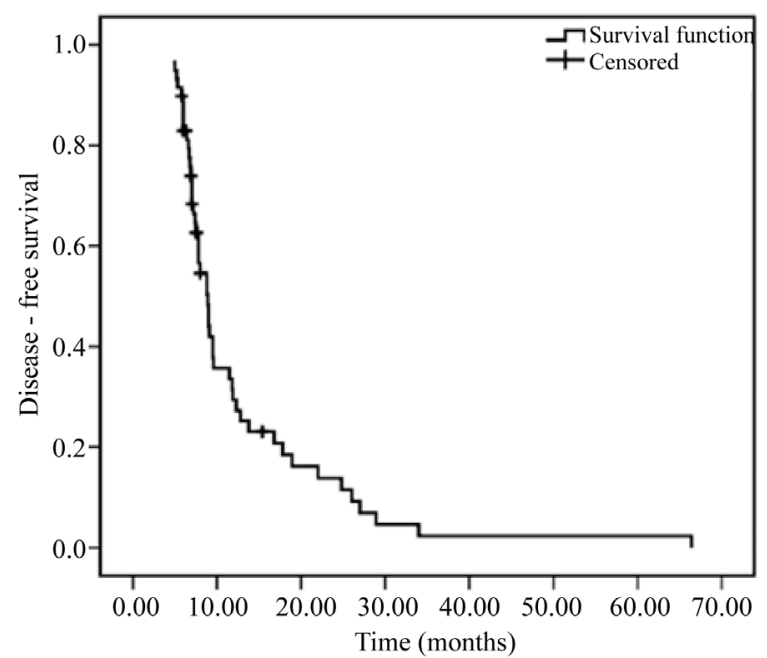

(a)

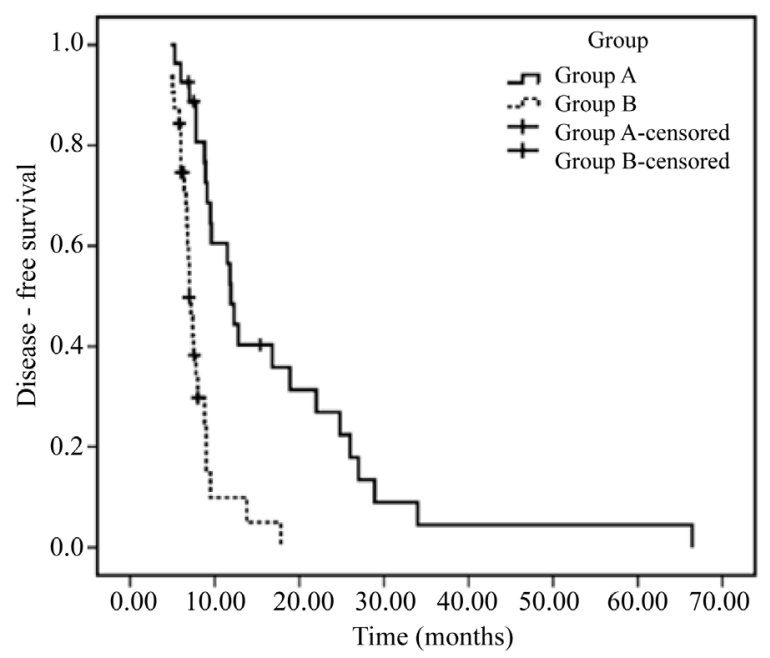

(c)

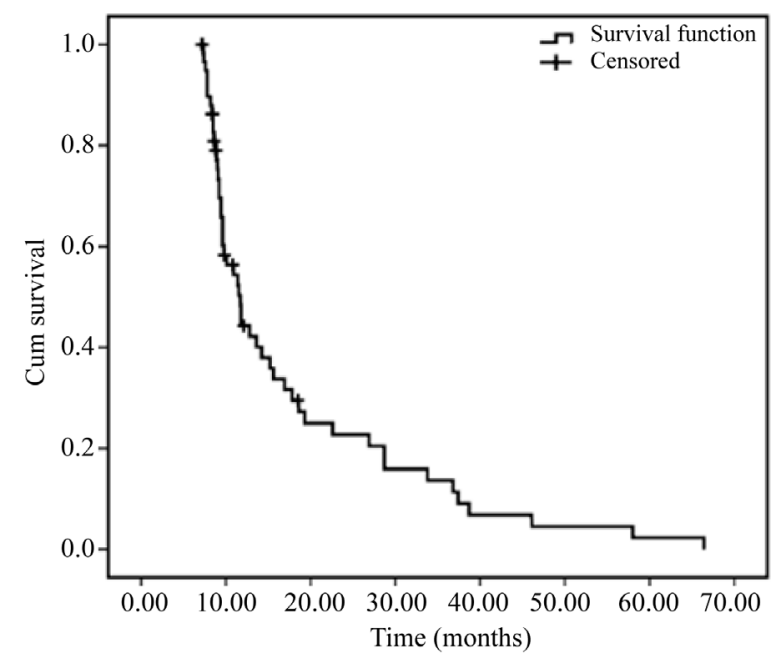

(b)

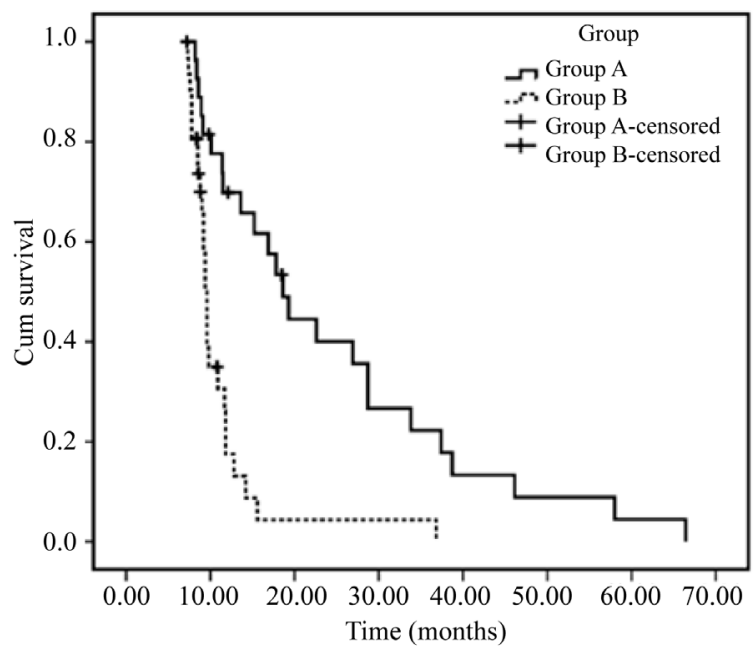

(d)

Figure 2. (a-b) Kaplan-Meier analysis of disease-free survival (DFS, a) and overall survival (OS, b) in all patients ( $\mathrm{n}=59$ ) with huge-size HCC who received SBRT following TACE. (c-d) Multivariate analysis of DFS (c) and OS (d) between group A (70\% isodose curve encompassing $\geq 60 \%$ of entire tumor) vs. group B (70\% isodose curve encompassing $<60 \%$ of entire tumor).

that normal tissues were irradiated were shown in Table 4. The complications and toxicities of the patients were shown in Table 5. No severe radiation-induced liver disease (RILD) observed during the median follow-up period of 18.4 months (range, 4 - 70 months). Grade 1 - 2 of liver and gastrointestinal AE were observed in 3 (5.1\%) and 5 (8.5\%) of 59 patients, respectively, while no > grade 3 AE was observed. Fatigue was the major complication observed in $40.7 \%$ (24/59) patients. The symptoms of these patients either disappeared spontaneously or were manageable. The dermatitis represented another common complication, and 2 (3.4\%) of 59 patients experienced grade 3 dermatitis that was difficult to be managed. It is noteworthy that both these two patients had extremely large tumors (i.e., $16.8 \mathrm{~cm}$ and $17.6 \mathrm{~cm}$, respectively). that were therefore very close to skin. Thus, these findings indicate that $\gamma$-ray SBRT following incomplete TACE is safe in the treatment of patients with huge-size unresectable HCC.

\section{Discussion}

Currently, treatment options for huge-size ( $\geq 10 \mathrm{~cm}$ in diameter) HCC are limited. Disease with large tumor is most likely to recur after treatment [26]-[28], probably due to unrecognized small-vessel tumor invasion [29]. 
Table 4. Maximal doses that normal tissue is irradiated.

\begin{tabular}{ccccccc}
\hline & \multicolumn{3}{c}{ Group A } & \multicolumn{3}{c}{ Group B } \\
\cline { 2 - 7 } Dose (Gy) & s.c. tissue & Stomach & Duodenum & s.c. tissue & Stomach & Duodenum \\
\hline Fractional dose (max) & 3.0 & 2.3 & 2.1 & 3.0 & 2.5 & 2.3 \\
Total dose (max) & 42 & 32 & 27.2 & 42 & 32.5 & 29.9 \\
\hline
\end{tabular}

s.c., subcutaneous; max, maximum.

Table 5. SBRT-related adverse events (AEs).

\begin{tabular}{|c|c|c|c|c|c|c|}
\hline \multirow{2}{*}{$\mathrm{AE}$} & \multicolumn{3}{|c|}{ Group A, no. of patient (\%) } & \multicolumn{3}{|c|}{ Group B, no. of patient (\%) } \\
\hline & Grade 1 & Grade 2 & Grade 3 & Grade 1 & Grade 2 & Grade 3 \\
\hline Edema & $1(3.7)$ & 0 & 0 & $1(3.1)$ & 0 & 0 \\
\hline Anemia & $1(3.7)$ & 0 & 0 & 0 & 0 & 0 \\
\hline GI & $1(3.7)$ & $1(3.7)$ & 0 & $2(6.2)$ & $1(3.1)$ & 0 \\
\hline Fatigue & $6(22.2)$ & $4(14.8)$ & 0 & $9(28.1)$ & $5(15.6)$ & 0 \\
\hline Nausea & $2(7.4)$ & $1(3.7)$ & 0 & $3(9.4)$ & $2(6.3)$ & 0 \\
\hline Dermatitis & $2(7.4)$ & $5(18.5)$ & $1(3.7)$ & $3(9.4)$ & $8(25)$ & $1(3.1)$ \\
\hline Liver function & $1(3.7)$ & 0 & 0 & $1(3.1)$ & $1(3.1)$ & 0 \\
\hline
\end{tabular}

GI, gastrointestinal.

Large tumor usually portends an unfavorable factor for patient prognosis [30]. Among others, the recently advanced radiotherapy represents a promising option for the treatment of patients with large-size HCC. In this context, our previous work has demonstrated the rationale for combining SBRT and TACE in treatment of HCC [21]. However, it remains unclear whether this combination radiotherapy could also benefit patients with large HCC tumors. To this end, clinical outcomes of 59 patients with huge-size unresectable HCC who had received SBRT following incomplete TACE were analyzed in the present study. Notably, after treated with this combination radiotherapy, $81.4 \%$ of patients with huge-size unresectable HCC achieved objective tumor responses, including 8.5\% CR and 72.9\% PR, while the ORR was much higher than those (29\%, including 4.3\% CD and 24.6\% $\mathrm{PR}$ ) reported very recently for TACE alone in treatment of huge-size HCC [31].

Of note, the dose prescription used in this study was different from other SBRT approaches for treatment of HCC [32]. The doses at $50 \%$ or $55 \%$ isodose curve by which the entire PTV was encompassed were normalized, while the higher isodose curves (e.g., $60 \%, 70 \%$, and $80 \%$ ) were all remained in the GTV. In addition, the range of $\mathrm{P}_{70}$ was $38 \%-72 \%$. These modifications yielded a much higher dose in GTV than in normal tissue around the tumor. Therefore, the high-dose in GTV is most likely responsible for the high ORR observed in this study such a high ORR.

It has been reported the survival rates of HCC patients treated with SBRT are similar to those treated with charged particle therapy [33]. The present study provide first evidence that the modality combining SBRT with TACE markedly prolonged overall survival of patients with large tumor HCC, with median OS of 11.8 months that almost doubled when compared to TACE alone (median OS = 6.5 months) [31]. Moreover, the present results also raise a notion that the higher dose-volume might be more beneficial to patient survival. Multivariate analysis indicated that while $\mathrm{P}_{70}, \mathrm{P}_{80}$, fractional dose, and Child-Pugh class were associated with DFS and OS, $\mathrm{P}_{70}$ is the only one statistically significant factors to predict patient survival. To this context, $\mathrm{P}_{70} \geq 60 \%$ yielded 1-, 3-, 5-year OS rates as $69.7 \%, 23.6 \%$, and $6.1 \%$, respectively, with the median OS of 18.3 months, comparable to those $(37.8 \%, 16.3 \%$, and 9.7\%) reported recently for TACE alone [34]. Consistent with the results that a higher ORR was achieved with larger $\mathrm{P}_{70}$, the difference in patient survival between two levels of dose-volume parameters (i.e., $\mathrm{P}_{70} \geq 60 \%$ versus $\mathrm{P}_{70}<60 \%$ ) indicates that the high dose-volume (for $\mathrm{P}_{70}$ in particular) played an important role in outcomes of patients with huge-size unresectable HCC after received SBRT. 
Both severe gastrointestinal AE and radiation-induced liver disease (RILD) have been reported after treatment of HCC with volumetric modulated arc therapy [35]. In contrast, neither severe (grade $\geq 3$ ) RILD nor gastrointestinal AE was observed in the present study. The major toxicities included fatigue and grade 1 - 2 dermatitis, while grade 3 dermatitis was seen only in 3.4\% (2/59) patients at 3 - 4 months after treatment with SBRT. It is noteworthy that both of these two patients who experienced grade 3 dermatitis had extremely large tumors that were $>16 \mathrm{~cm}$ in diameter, as well as very close to skin. In the present study, all patients received daily fraction of 2.6 - 3.0 Gy with 6 fractions per week for 12 - 14 days, these fractional doses are higher than the conventional radiotherapy. Thus, severe dermatitis might be related to the high dose radiation, which it raises a possibility that dose or time of SBRT might need to be optimized to avoid such a severe adverse event (AE) in treatment of very large HCC tumor (e.g., >16 cm), particularly when tumor is near skin. In this context, it has been suggested that administration of radiotherapy for a shorter period of time might be more appropriate for treatment of HCC patients [36]. Nevertheless, although it is rare, severe dermatitis should be taken into consideration to treat extremely large HCC tumors with high dose-volume SBRT.

\section{Conclusion}

Together, these results suggest that the modality combining SBRT and TACE is a safe and effective in the treatment of patients with huge-size unresectable HCC. It is noteworthy that among several key factors tested, the dose-volume $\mathrm{P}_{70}$ is the only one parameter that is able to statistically significantly predict better survival (e.g., longer DFS and OS) in this population of patients who receive SBRT and TACE in combination. Therefore, high dose-volume (e.g., $\mathrm{P}_{70} \geq 60 \%$ ) plays an important role in efficacy of this regimen. However, there are certain limitations in this study due to the nature of a retrospective study. In addition, the percentage of tumor volume in entire liver is not taken into account in this study. Therefore, although future perspective randomized studies are required to define efficacy and safety of SBRT combined with TACE, the promising patient outcomes observed in this study argue strongly that this combination radiotherapy warrants further attention in the treatment of patients with huge-size unresectable HCC.

\section{References}

[1] Ferlay, J., Soerjomataram, I., Dikshit, R., et al. (2015) Cancer Incidence and Mortality Worldwide: Sources, Methods and Major Patterns in GLOBOCAN 2012. International Journal of Cancer, 136, E359-E386. http://dx.doi.org/10.1002/ijc.29210

[2] Sangiovanni, A., Del Ninno, E., Fasani, P., et al. (2004) Increased Survival of Cirrhotic Patient with a Hepatocellular Carcinoma Detected during Surveillance. Gastroenterology, 126, 1005-1014. http://dx.doi.org/10.1053/j.gastro.2003.12.049

[3] Thomas, M.B., Jaffe, D., Choti, M.M., et al. (2010) Hepatocellular Carcinoma: Consensus Recommendations of the National Cancer Institute Clinical Trials Planning Meeting. Journal of Clinical Oncology, 28, 3994-4005. http://dx.doi.org/10.1200/JCO.2010.28.7805

[4] Forner, A., Llovet, J.M. and Bruix, J. (2012) Hepatocellular Carcinoma. The Lancet, 379, 1245-1255. http://dx.doi.org/10.1016/S0140-6736(11)61347-0

[5] The Liver Cancer Group of Japan (1994) Predictive Factors for Long Term Prognosis after Partial Hepatectomy for Patients with Hepatocellular Carcinoma in Japan. Cancer, 74, 2772-2780. http://dx.doi.org/10.1002/1097-0142(19941115)74:10<2772::AID-CNCR2820741006>3.0.CO;2-V

[6] Bruix, J. and Sherman, M. (2011) Management of Hepatocellular Carcinoma: An Update. Hepatology, 53, $1020-1022$. http://dx.doi.org/10.1002/hep.24199

[7] Chirica, M., Scatton, O., Massault, P.P., et al. (2008) Treatment of Stage IVA Hepatocellular Carcinoma: Should We Reappraise the Role of Surgery? Archives of surgery, 143, 538-543. http://dx.doi.org/10.1001/archsurg.143.6.538

[8] Attwa, M.H. and El-Etreby, S.A. (2015) Guide for Diagnosis and Treatment of Hepatocellular Carcinoma. World Journal of Hepatology, 7, 1632-1651. http://dx.doi.org/10.4254/wjh.v7.i12.1632

[9] Trevisani, F., De Notariis, S., Rossi, C. and Bernardi. M. (2001) Randomized Control Trials on Chemoembolization for Hepatocellular Carcinoma: Is There Room for New Studies? Journal of Clinical Gastroenterology, 32, 383-389. http://dx.doi.org/10.1097/00004836-200105000-00005

[10] Wang, J.H., Kuo, Y.H., Wang, C.C., et al. (2013) Surgical Resection Improves the Survival of Selected Hepatocellular Carcinoma Patients in Barcelona Clinic Liver Cancer Stage C. Digestive and Liver Disease, 45, 510-515. http://dx.doi.org/10.1016/j.dld.2012.10.019 
[11] Morise, Z., Kawabe, N., Tomishige, H., et al. (2014) Recent Advances in the Surgical Treatment of Hepatocellular Carcinoma. World Journal of Gastroenterology, 20, 14381-14392. http://dx.doi.org/10.3748/wjg.v20.i39.14381

[12] Chen, J.H., Wei, C.K., Lee, C.H., Chang, C.M., Hsu, T.W. and Yin, W.Y. (2015) The Safety and Adequacy of Resection on Hepatocellular Carcinoma Larger than $10 \mathrm{~cm}$ : A Retrospective Study over 10 Years. Annals of Medicine and Surgery, 4, 193-199. http://dx.doi.org/10.1016/j.amsu.2015.05.003

[13] Dahiya, D., Wu, T.J., Lee, C.F., Chan, K.M., Lee, W.C. and Chen, M.F. (2010) Minor versus Major Hepatic Resection for Small Hepatocellular Carcinoma (HCC) in Cirrhotic Patients: A 20-Year Experience. Surgery, 147, $676-685$. http://dx.doi.org/10.1016/j.surg.2009.10.043

[14] Guerrero-Misas, M., Rodríguez-Perálvarez, M. and De la Mata, M. (2015) Strategies to Improve Outcome of Patients with Hepatocellular Carcinoma Receiving a Liver Transplantation. World Journal of Hepatology, 7, 649-661. http://dx.doi.org/10.4254/wjh.v7.i4.649

[15] Cochrane, A.M.G., Murray-Lyon, I.M., Brinkley, D.M. and Williams, R. (1977) Quadruple Chemotherapy versus Radiotherapy in Treatment of Primary Hepatocellular Carcinoma. Cancer, 40, 609-614. http://dx.doi.org/10.1002/1097-0142(197708)40:2<609::AID-CNCR2820400203>3.0.CO;2-L

[16] Lewin, K. and Millis, R.R. (1973) Human Radiation Hepatitis. A Morphologic Study with Emphasis on the Late Changes. Archives of Pathology, 96, 21-26.

[17] Lawrence, T.S., Robertson, J.M., Anscher, M.S., Jirtle, R.L., Ensminger, W.D. and Fajardo, L.F. (1995) Hepatic Toxicity Resulting from Cancer Treatment. International Journal of Radiation Oncology *Biology* Physics, 31, 1237 1248. http://dx.doi.org/10.1016/0360-3016(94)00418-K

[18] Gomaa, A.I. and Waked, I. (2015) Recent Advances in Multidisciplinary Management of Hepatocellular Carcinoma. World Journal of Hepatology, 7, 673-687. http://dx.doi.org/10.4254/wjh.v7.i4.673

[19] Price, T.R., Perkins, S.M., Sandrasegaran, K., et al. (2012) Evaluation of Response after Stereotactic Body Radiotherapy for Hepatocellular Carcinoma. Cancer, 118, 3191-3198. http://dx.doi.org/10.1002/cncr.26404

[20] Zhong, N.B., Chen, Z.H. and Lv, G.M. (2013) A Clinical Study on Stereotactic Body Radiotherapy for Hepatocellular Carcinoma with Portal Vein Tumor Thrombosis. Journal of Cancer Research and Therapeutics, 1, 143-148. http://dx.doi.org/10.14312/2052-4994.2013-22

[21] Zhong, N.B., Lv, G.M. and Chen, Z.H. (2014) Stereotactic Body Radiotherapy Combined with Transarterial Chemoembolization for Huge $(\geq 10 \mathrm{~cm})$ Hepatocellular Carcinoma: A Clinical Study. Molecular and Clinical Oncology, 2, 839-844.

[22] Tse, R.V., Hawkins, M., Lockwood, G., et al. (2008) Phase I Study of Individualized Stereotactic Body Radiotherapy for Hepatocellular Carcinoma and Intrahepatic Cholangiocarcinoma. Journal of Clinical Oncology, 26, 657-664. http://dx.doi.org/10.1200/JCO.2007.14.3529

[23] Van De Voorde, L., Vanneste, B., Houben, R., et al. (2015) Image-Guided Stereotactic Ablative Radiotherapy for the Liver: A Safe and Effective Treatment. European Journal of Surgical Oncology, 41, 249-256. http://dx.doi.org/10.1016/j.ejso.2014.10.053

[24] Culleton, S., Jiang, H., Haddad, C.R., et al. (2014) Outcomes Following Definitive Stereotactic Body Radiotherapy for Patients with Child-Pugh B or C Hepatocellular Carcinoma. Radiotherapy and Oncology, 111, 412-417. http://dx.doi.org/10.1016/j.radonc.2014.05.002

[25] Scorsetti, M., Comito, T., Cozzi, L., et al. (2015) The Challenge of Inoperable Hepatocellular Carcinoma (HCC): Results of a Single-Institutional Experience on Stereotactic Body Radiation Therapy (SBRT). Journal of Cancer Research and Clinical Oncology, 141, 1301-1309. http://dx.doi.org/10.1007/s00432-015-1929-y

[26] Shah, S.A., Greig, P.D., Galllinger, S., et al. (2006) Factors Associated with Early Recurrence after Resection for Hepatocellular Carcinoma and Outcomes. Journal of the American College of Surgeons, 202, 275-283. http://dx.doi.org/10.1016/j.jamcollsurg.2005.10.005

[27] Regimbeau, J.M., Abdalla, E.K., Vauthey, J.N., et al. (2004) Risk Factors for Early Death Due to Recurrence after Liver Resection for Hepatocellular Carcinoma: Results of a Multicenter Study. Journal of Surgical Oncology, 85, 3641. http://dx.doi.org/10.1002/jso.10284

[28] Yamamoto, Y., Ikoma, H., Morimura, R., et al. (2015) Optimal Duration of the Early and Late Recurrence of Hepatocellular Carcinoma after Hepatectomy. World Journal of Gastroenterology, 21, 1207-1215. http://dx.doi.org/10.3748/wjg.v21.i4.1207

[29] Pawlik, T.M., Delman, K.A., Vauthey, J.N., et al. (2005) Tumor Size Predicts Vascular Invasion and Histologic Grade: Implications for Selection of Surgical Treatment for Hepatocellular Carcinoma. Liver Transplantation, 11, $1086-1092$. http://dx.doi.org/10.1002/lt.20472

[30] Llovet, J.M., Schwartz, M. and Mazzaferro, V. (2005) Resection and Liver Transplantation for Hepatocellular Carcinoma. Seminars in Liver Disease, 25, 181-200. http://dx.doi.org/10.1055/s-2005-871198 
[31] Xue, T., Le, F., Chen, R., et al. (2015) Transarterial Chemoembolization for Huge Hepatocellular Carcinoma with Diameter over Ten Centimeters: A Large Cohort Study. Medical Oncology, 32, 64. http://dx.doi.org/10.1007/s12032-015-0504-3

[32] Eriguchi, T., Takeda, A., Oku, Y., et al. (2013) Multi-Institutional Comparison of Treatment Planning Using Stereotactic Ablative Body Radiotherapy for Hepatocellular Carcinoma-Benchmark for a Prospective Multi-Institutional Study. Radiation Oncology, 8, 113. http://dx.doi.org/10.1186/1748-717X-8-113

[33] Qi, W.X., Fu, S., Zhang, Q. and Guo, X.M. (2015) Charged Particle Therapy versus Photon Therapy for Patients with Hepatocellular Carcinoma: A Systematic Review and Meta-Analysis. Radiotherapy and Oncology, 114, $289-295$. http://dx.doi.org/10.1016/j.radonc.2014.11.033

[34] Min, Y.W., Lee, J.H., Gwak, G.Y., et al. (2014) Long-Term Survival after Surgical Resection for Huge Hepatocellular Carcinoma: Comparison with Transarterial Chemoembolization after Propensity Score Matching. Journal of Gastroenterology and Hepatology, 29, 1043-1048. http://dx.doi.org/10.1111/jgh.12504

[35] Wang, P.M., Hsu, W.C., Chung, N.N., Chang, F.L., Fogliata, A. and Cozzi, L. (2012) Radiation Treatment with Volumetric Modulated Arc Therapy of Hepatocellular Carcinoma Patients. Early Clinical Outcome and Toxicity Profile from a Retrospective Analysis of 138 Patients. Radiation Oncology, 7, 207. http://dx.doi.org/10.1186/1748-717X-7-207

[36] Toya, R., Murakami, R., Baba, Y., et al. (2007) Conformal Radiotherapy for Portal Vein Tumor THROMBOSIS of Hepatocellular Carcinoma. Radiotherapy and Oncology, 84, 266-271. http://dx.doi.org/10.1016/j.radonc.2007.07.005 